\title{
Subespecificación y monoptongación en los sufijos de primera y segunda persona poseedora en el quechua de Chachapoyas
}

\section{Underspecification and monophthongization in the first and second possessive markers in Chachapoyas Quechua}

\author{
Jairo Valqui \\ Universidad Nacional Mayor de San Marcos \\ Contacto: jvalquic@unmsm.edu.pe \\ Gustavo Solís \\ Universidad Nacional Mayor de San Marcos \\ gsolisf@unmsm.edu.pe \\ Carlos Faucet \\ Universidad Nacional Mayor de San Marcos \\ carlos.faucet@unmsm.edu.pe \\ Franklin Espinoza \\ Universidad Nacional Mayor de San Marcos \\ franklin.espinoza@unmsm.edu.pe \\ Liz Velásquez \\ Universidad Nacional Mayor de San Marcos \\ liz.velasquez@unmsm.edu.pe
}

\section{Resumen}

En este artículo se discute el surgimiento de vocales monoptongadas breves y alargadas en el contexto de los sufijos de primera y segunda persona poseedora del quechua de Chachapoyas. La literatura previa aduce que el segmento inicial /-y/ de ambos sufijos explica la altura de las vocales y que el acento es el responsable de las alternancias entre vocales breves y largas: [i]/[i:] y [e]/[e:]. Sin embargo, la evidencia acústica permite proponer una nueva representación del segmento inicial de las personas poseedoras a través de un segmento subespecificado con el rasgo [coronal], así como explicar la alternancia entre las vocales monoptongadas breves y largas en el campo de la fonología léxica. Finalmente, se deriva también algunos comentarios acerca del cambio histórico del marcador de primera persona en otras variedades de quechua.

Palabras clave: Monoptongación; subespecificación; fonología léxica; persona poseedora; quechua de Chachapoyas 


\begin{abstract}
This paper discusses on the emergence of long and short monophthongized vowels in a number of suffixes of the Quechua variety spoken in Chachapoyas. In particular, vowel monophthongization occurs within the possessive suffixes that mark first and second person in this Quechua variety. Previous studies have explained that alternations between $[\mathrm{i}] /[\mathrm{i}:]$ and $[\mathrm{e}] /[\mathrm{e}:]$ are the result of variations in the quality of the initial segment /-y/ that appears in both first and second person possessive suffixes. Similarly, previous research has described the alternation between short and long vowel segments (i.e. [i]/[i:] and $[\mathrm{e}] /[\mathrm{e}]])$ as the outcome of stress variation. However, recent acoustic evidence suggests that the initial segment of both possessive suffixes is not /-y/ but a subespecified coronal segment. Moreover, this evidence allows to explain the alternation between short and long vowels as caused by the lexical phonology of Chachapoyas Quechua rather than as a result of stress variation. After discussing these new findings, this paper provides some insights about the linguistic change of the first person possessive suffix in other Quechua varieties.
\end{abstract}

Palabras clave: Monophthongization (Fusion); Underspecification; Lexical Phonology; Possessive Person Markers; Chachapoyas Quechua

\title{
Introducción
}

La variedad quechua de Chachapoyas es considerada, según el nivel de vitalidad de una lengua (Unesco, 2003), como una variedad en situación crítica. Es decir, una variedad lingüística que es usada por muy pocos hablantes de la generación de los abuelos. El escaso conocimiento que se tiene sobre esta variedad se debe, principalmente, a la contribución del lingüista Gerald Taylor, quien empezó a realizar estudios en la zona a fines de la década del 60. Según sus publicaciones, Taylor $(1994,2000)$ registró datos de distintos pueblos de las provincias de Chachapoyas y Luya que conformarían esta variedad denominada "quechua de Chachapoyas"1. Otros trabajos relacionados con esta variedad fueron publicados por Carmelo Chaparro (1985), realizado en los pueblos de Granada, Olleros y Quinjalca en el Alto Imaza (Chachapoyas), y por David Weber (1974) en forma de apuntes de investigación del quechua de Lamud (Luya).

Según el reporte de Taylor (1994), en la segunda parte del siglo pasado era posible encontrar hablantes de esta variedad quechua: muy pocos en pueblos cercanos a la capital, pero muchos más en zonas más alejadas como en Luya o La Jalca e incluso monolingües en los pueblos del Alto Imaza. Esa realidad ha cambiado notablemente. En trabajos de campo realizados entre los años 20152016, se ha podido reportar poco más de una decena de hablantes fluidos de esta 
variedad en su totalidad pertenecientes a la generación adulta que solo usan la lengua en ocasiones esporádicas, ahora solo cuando algún visitante les solicita alguna información en esta variedad del quechua.

De acuerdo a algunas características lingüísticas, esta variedad fue emparentada con otros sistemas quechuas dentro del QII (Cf. Torero, 2002; Cerrón-Palomino, 2003). Sin embargo, algunos procesos fonológicos como el que trataremos enseguida permiten abrir una discusión en torno a este tema. Fonológicamente, la variedad quechua de Chachapoyas presenta un inventario de 18 unidades segmentales. De acuerdo con las propuestas de Taylor (1994, 2000, 2006), el sistema consonántico está compuesto por 15 segmentos, como se muestra en (1).

(1) Sistema de consonantes del quechua de Chachapoyas

\begin{tabular}{|l|c|c|c|l|l|l|}
\hline & $\begin{array}{l}\text { Bilabia- } \\
\text { les }\end{array}$ & Dentales & Alveolares & $\begin{array}{l}\text { Palata- } \\
\text { les }\end{array}$ & $\begin{array}{l}\text { Retrofle- } \\
\text { jas }\end{array}$ & Velares \\
\hline Oclusivas & $\mathrm{p}$ & $\mathrm{t}$ & & & & $\mathrm{k}$ \\
\hline Africadas & & & & $\mathrm{t} \int$ & $\mathrm{ts}$ & \\
\hline Fricativas & & & $\mathrm{s}$ & $\mathrm{J}$ & & \\
\hline Nasales & $\mathrm{m}$ & $\mathrm{n}$ & & $\mathrm{n}$ & & \\
\hline Laterales & & & $\mathrm{l}$ & $\kappa / \mathrm{d}^{2}$ & & \\
\hline Vibrante & & & $\mathrm{r}$ & & & \\
\hline Semivocales & $\mathrm{w}$ & & & $\mathrm{y}$ & & \\
\hline
\end{tabular}

A partir de Taylor (1994, p. 21; 2006, p. 13)

En el sistema vocálico, el autor propone 3 segmentos, como se muestra en (2). Según su altura, /i/ y /u/ son caracterizadas como altas, mientras que /a/ es baja; y según su localización, /i/ es definida como anterior, mientras que /a/ y $/ \mathrm{u} /$ como central y posterior, respectivamente.

(2) Sistema de vocales de la variedad quechua de Chachapoyas

\begin{tabular}{|l|c|l|c|}
\hline & Anterior & Central & Posterior \\
\hline Alta(s) & $\mathrm{i}$ & & $\mathrm{u}$ \\
\hline Baja & & $\mathrm{a}$ & \\
\hline
\end{tabular}

A partir de Taylor (1994, p. 22) 
En la descripción morfológica de las personas poseedoras, Taylor (2000) propone cuatro sufijos para indicar tres personas poseedoras en singular y una en plural. Junto a tales sufijos, el autor añade la secuencia /-NI-/ que representa, como él señala, un apoyo fonético que sigue a las raíces nominales que acaban en consonante, como se observa en (3).

$\begin{array}{ll}\text { /-Niy/ } & \text { 1.a pos. sing. } \\ \text { /-NIyki/ } & \text { 2.a pos. sing. } \\ \text { /-NIn/ } & \text { 3.a pos. sing. } \\ \text { /-NInt } \mathrm{i} / & \text { 1.a pos. pl. }\end{array}$

Fuente: (Taylor, 2000, p. 79)

Uno de los puntos problemáticos de esta propuesta se relaciona con la evidencia fonética del segmento /-y/ de los sufijos de primera y segunda persona poseedora cuando estos se añaden a raíces nominales, pues, como se puede observar en las figuras 1 y 2 , este segmento nunca aparece. Así, de los datos acústicos registrados en la Jalca Grande (Chachapoyas), si a una raíz nominal que termina en /a/ como /uma/ 'cabeza' se le añade el sufijo de 1.a persona singular, el resultado es una vocal media alargada ['ume:] 'mi cabeza', como se observa en la figura 1, y no ${ }^{\star}$ ['umay] como se podría esperar.

Figura 1. Espectrograma de la palabra [ume:]

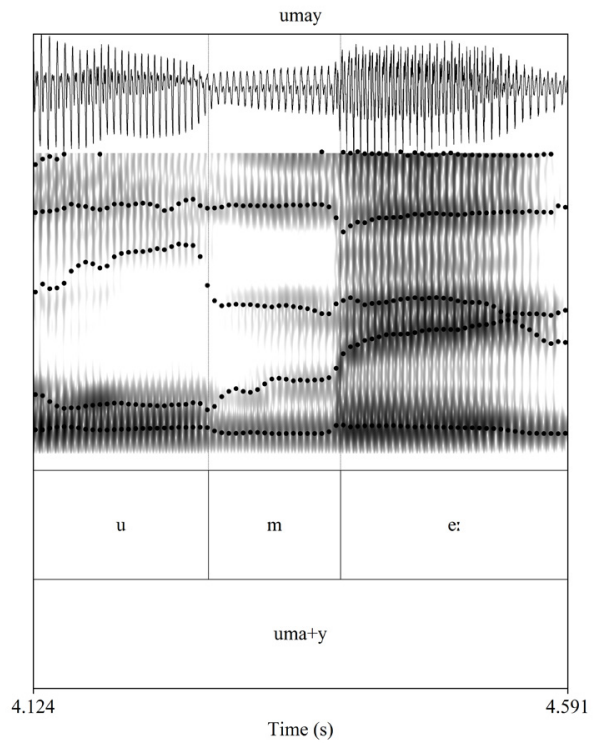


Así también, si a una raíz nominal que termina en /i/ o /u/, como / maki/ 'mano' o / SuNku/ 'corazón', se le añade el sufijo de 2.a persona poseedora, el resultado es una vocal alta breve ['makiki] 'tu mano' y ['Sungiki] 'tu corazón', respectivamente. En la figura 2, por ejemplo, se muestra el espectrograma de /maki/ + 2.a persona poseedora cuyo resultado es ['makiki] y no *['makiyki], sin la presencia del segmento en cuestión [-y].

Figura 2. Espectrograma de la palabra ['makiki]

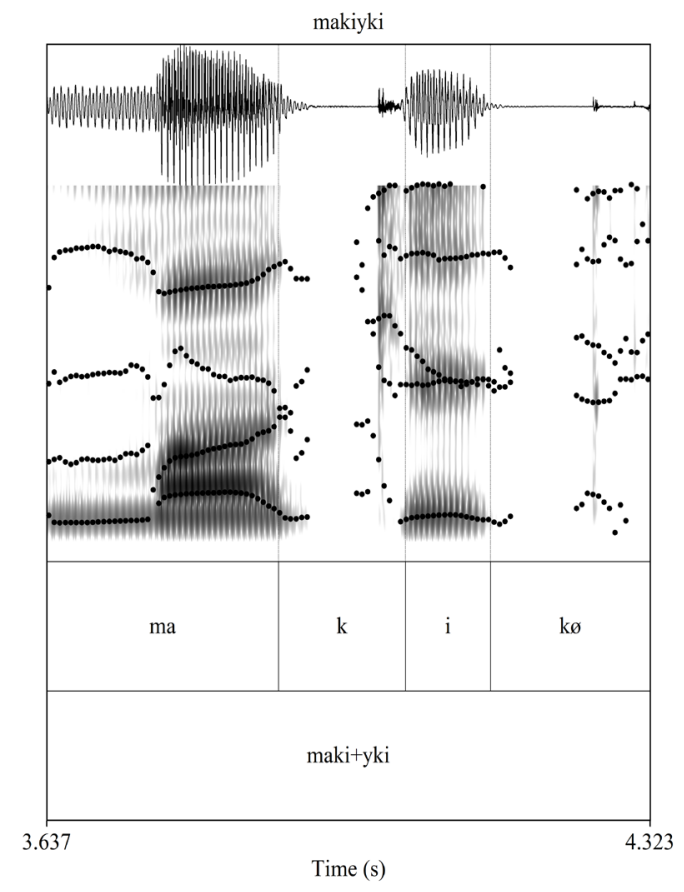

Si los sufijos de primera y segunda persona poseedora, de acuerdo a los datos acústicos reportados aquí, nunca exhiben el segmento /-y/ de la 1.a y 2. a persona poseedora, ¿por qué Taylor así lo propuso? Al parecer el hecho de que Taylor postule /-y/ como parte de estos sufijos se justificaría como explicación de la aparición de las vocales monoptongadas ${ }^{3}$ en la variedad en cuestión. Para Taylor (2000) solo la secuencia /a+y/ deviene en la vocal monoptongada ê: y las secuencias $/ \mathrm{i}+\mathrm{y} / \mathrm{y} / \mathrm{u}+\mathrm{y} /$ en la vocal monoptongada $\hat{i}^{4}{ }^{4}$. Si bien es cierto que tal explicación resulta plausible en la medida en que existen variedades quechuas que evidencian este segmento y además se han reportado otras variedades que muestran un proceso similar en el cual la secuencia /ay/ genera formas como [ey] (cf. Quesada, 1998; Solis, 1996), en este 
trabajo proponemos otra explicación del fenómeno fonológico aquí observado. En esta perspectiva, la propuesta que se adelanta representa un segmento subespecificado del segmento inicial de los sufijos de primera y segunda persona poseedora en el quechua de Chachapoyas. Esta forma subespecificada solo con el rasgo [coronal] en el punto de articulación, [-abierto] en la altura, y una unidad de tiempo $(\mu)$ al someterse a un proceso de fusión (monoptongación), genera las formas atestiguadas acústicamente en esta variedad quechua.

Para argumentar desde esta perspectiva, el artículo se divide en 4 secciones. En $\$ 1$ se presenta la teoría de partículas propuesta por Schane (1995), donde se sostiene que los procesos de monoptongación son reglas de fusión que operan en las lenguas del mundo. En $\$ 2$ se explicita la metodología para el recojo de la data y para el análisis de esta. En $\$ 3$ damos cuenta de los resultados acústicos de las vocales simples y de las monoptongadas, así como también de los contextos de aparición de estas. Finalmente, en $\$ 4$ discutimos el surgimiento de las secuencias vocálicas monoptongadas desde la perspectiva asumida en este trabajo y sus posibles desarrollos en el contexto mayor de las variedades quechua modernas.

\section{Marco teórico}

De acuerdo con Schane (1995), la monoptongación es un proceso fonológico que fusiona una secuencia de partículas (rasgos) en una sola. Las partículas intervinientes son la de apertura, relacionada con la altura vocálica, y la de tonalidad, representadas por $|\mathrm{a}|$ y por $|\mathrm{y}|$, respectivamente. La última puede ser subdividida, a su vez, en palatalidad y labialidad, representadas por $|\mathrm{i}| \mathrm{y}|\mathrm{u}|$, como se muestra en la figura 3.

Figura 3. Representación de las partículas en la teoría de Schane (1995)

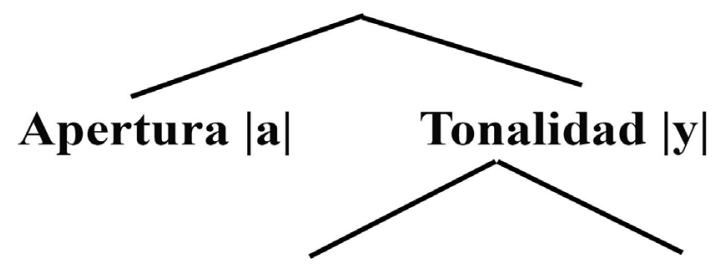

Palatalidad $|\mathbf{i}| \quad$ Labialidad $|\mathbf{u}|$

Fuente: (Shane, 1995, p. 592) 
Estas partículas pueden estar asociadas a distintas raíces, como se muestra en la figura (4.a), o compartir alguna, como se muestra en (4.b). Si estas se fusionan, el resultado es una configuración como la de (4.c).

Figura 4. Distintas configuraciones al fusionarse las partículas

(a)

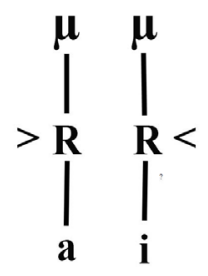

[aij] (b)

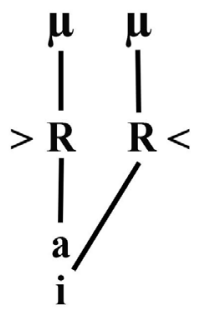

[ei]] (c)

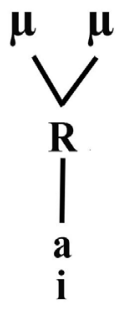

[e:]

Fuente: (Shane, 1995, p. 592)

En la configuración (4.c), la fusión combina dos nudos de raíz en uno solo. En ese sentido, el proceso de monoptongación es entendido como la fusión de los nudos R de un diptongo en un solo nudo R completamente superpuesto. El resultado es una nueva configuración con un solo nudo $\mathrm{R}$ asociado a dos unidades de tiempo, el que es representado aquí por moras $(\mu)$. Sin embargo, según advierte Schane (1995), el proceso de fusión también ocurriría en niveles superiores a los rasgos: puede afectar a las raíces $>\mathrm{RR}<$, a las unidades de tiempo $>\mu \mu<\mathrm{o}$ a las sílabas $>\sigma \sigma<$.

Debido a que este trabajo trata sobre la diptongación y la monoptongación, los procesos de fisión y fusión han actuado principalmente en los nodos raíz. Estos procesos, sin embargo, resultan ser más generales. No están restringidos solo a las raíces. Cualquier nodo sobre la raíz es un candidato para la fisión / fusión. [...], la fusión de dos nudos $\mu$ convertirá una vocal larga en una corta, o un diptongo decreciente en uno creciente o acortado. (Schane, 1995, pp. 604-605, traducción nuestra)

En (4) se muestra la notación para un proceso de monoptongación que afecta al nivel de la raíz (R) del segmento melódico. Como se puede observar ahí, la fusión combina dos nudos de raíz en uno solo. Siendo así, el proceso de monoptongación sería la fusión de los nudos $\mathrm{R}$ de una secuencia de vocales en un solo nudo R completamente unificados. El resultado es una nueva configura- 
ción con un solo nudo $\mathrm{R}$ asociado a 2 unidades de tiempo $(\mu)$, como se propone para el surgimiento de las vocales [e:] y [o:] en sánscrito (Schane, 1995). Por otra parte, en (5), se representa la fusión al nivel de nudos de tiempo $(\mu)$, es decir, en un nivel superior a la raíz $(\mathrm{R})$, como ocurre al pasar de diptongo decreciente con dos moras a uno creciente con una sola mora (Schane, 1995):
(4)
$\mu \mu,>\mathrm{RR}<$
$\left[\varnothing_{12}, a_{1}, y_{2}\right]$
(5)

$>\mu \mu<, \mathrm{RR} \quad\left[\varnothing_{12}\right.$, ia $\left._{1}, \mathrm{y}_{2}\right]$
$[\mathrm{eu}]>[$ io $]$

\section{Metodología}

El presente trabajo es considerado de corte descriptivo-explicativo. El objetivo principal fue dar cuenta de las vocales monoptongadas [e] e [i] en el quechua de Chachapoyas. Para este fin, primero se recogió datos acústicos de las vocales simples [i], [u], [a] y de las vocales monoptongadas [e] e [i]; segundo, se analizó los contextos de aparición de las vocales monoptongadas. Los datos utilizados corresponden a la Jalca Grande en la provincia de Chachapoyas (número 3 en la figura 5) y fueron obtenidos como parte del proyecto de investigación denominado "Documentación lingüística de eventos comunicativos en el quechua de Chachapoyas" financiado por la Universidad Nacional Mayor de San Marcos con código 160304051.

Figura 5. Distribución espacial de los pueblos donde se registraron los datos

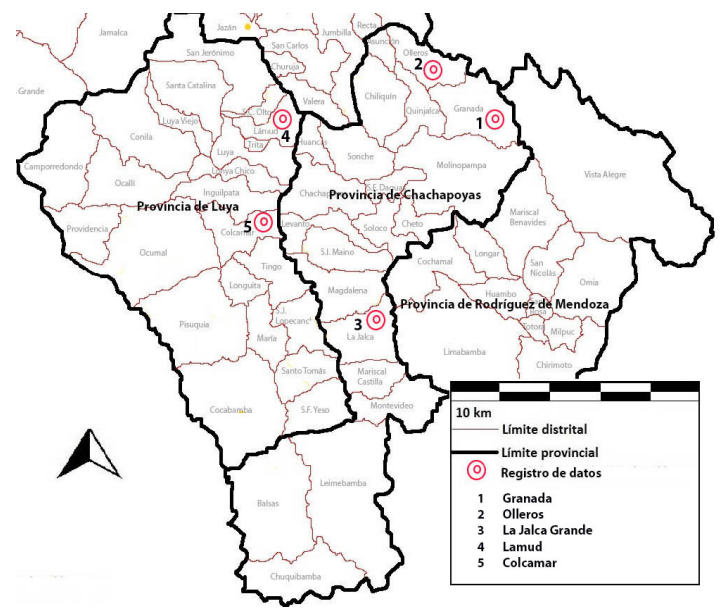


Para el recojo de la data se utilizó la lista de ciento cincuenta significados de relevancia cultural y lingüística en los Andes propuesta por Heggarty (2005) con la finalidad de registrar las vocales que aparecían en las raíces quechuas. Luego se indujo la elicitación de las raíces nominales de la lista más los sufijos de 1.a y 2.a persona poseedora. Los datos aquí presentados pertenecen a uno de los pocos hablantes de esta variedad quechua, quien fue codificado como IC. Las grabaciones fueron registradas en formato de alta calidad en formato wav mediante una grabadora digital Tascam DR-40 y un micrófono Shure WH30XLR.

Para el análisis acústico se consideró, por una parte, la medición de los formantes F1 y F2 de las vocales [i], [u] y [a] dentro de las raíces nominales y, por otra parte, de las vocales monoptongadas [e] e [i] cuando aparecen entre raíces y los sufijos de persona poseedora. Por ejemplo, en la figura 6, la flecha inferior muestra el F1 y la flecha superior el F2 de la vocal [i] en la palabra ['tşakiki] 'tu pie'.

Figura 6. F1 y F2 de la vocal [i] en posición media en la palabra ['tşakiki]

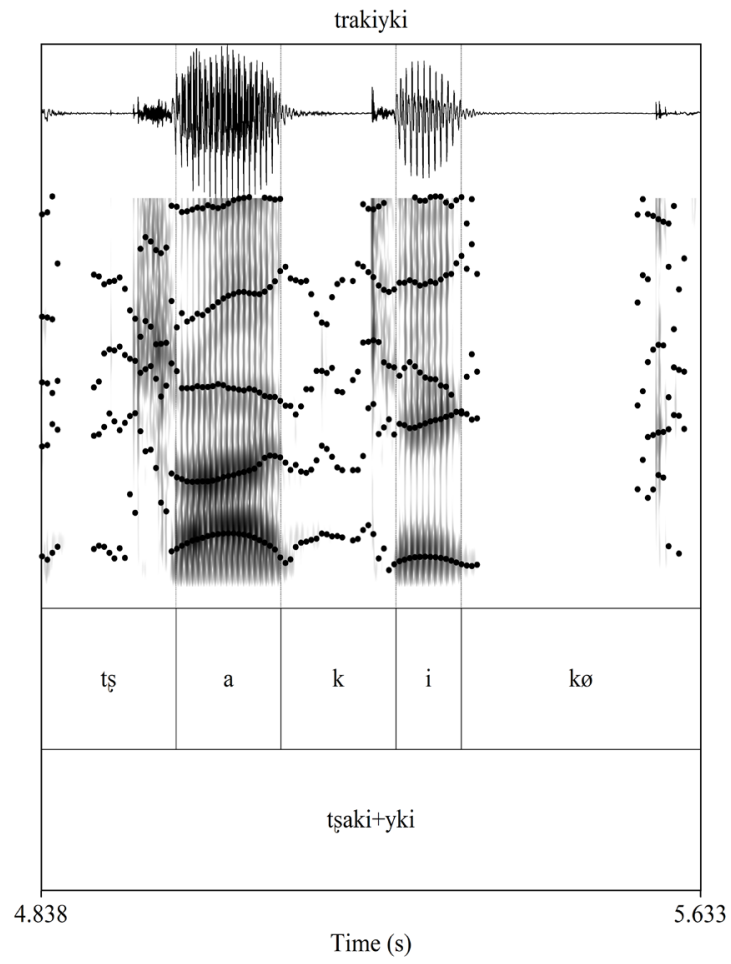




\section{Resultados}

\subsection{Medición de los formantes F1 y F2 de las vocales simples y monopton- gadas}

Como se ha presentado en la parte introductoria de este trabajo, el proceso en discusión evidencia que el contacto entre las vocales /a, i, u/ de las raíces nominales en el quechua de Chachapoyas más el primer segmento de la 1.a y 2.a persona poseedora genera vocales que se fusionan en algunos casos en [e] y en otros en [i]. En primer lugar, la medición de los formantes F1 y F2 muestra una dispersión de las emisiones fonéticas de [a, i, u] en 3 grandes espacios, como se observa en la figura 7. El promedio de la vocal simple y monoptongada [i] fue $342 \mathrm{~Hz}$ para el F1 y $2080 \mathrm{~Hz}$ para el F2; el de la vocal [u], $340 \mathrm{~Hz}$ para el F1 y 984 $\mathrm{Hz}$ para el F2; y el de la vocal [a], $589 \mathrm{~Hz}$ para el F1 y $1332 \mathrm{~Hz}$ para el F2.

Figura 7. Carta de formantes de la vocales [i], [u] y [a] en el quechua de Chachapoyas

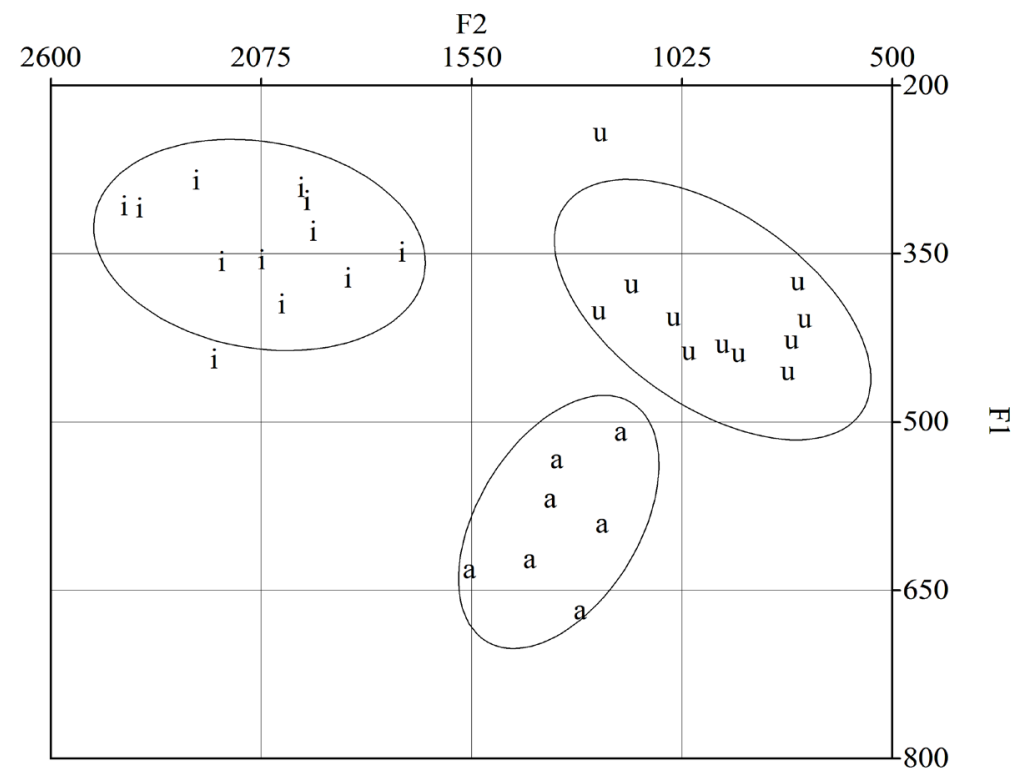

En segundo lugar, la medición de los formantes de la vocal monoptongada [e] permite ubicarla entre la dispersión de [i] y de la vocal [a] en frecuencias altas para el F2 según se observa en la figura 8. El promedio del F1 de esta vocal fue $477 \mathrm{~Hz}$ y el del F2, $1914 \mathrm{~Hz}$. 
Figura 8. Ubicación de la vocal [e] en la carta de formantes del quechua de Chachapoyas

F2

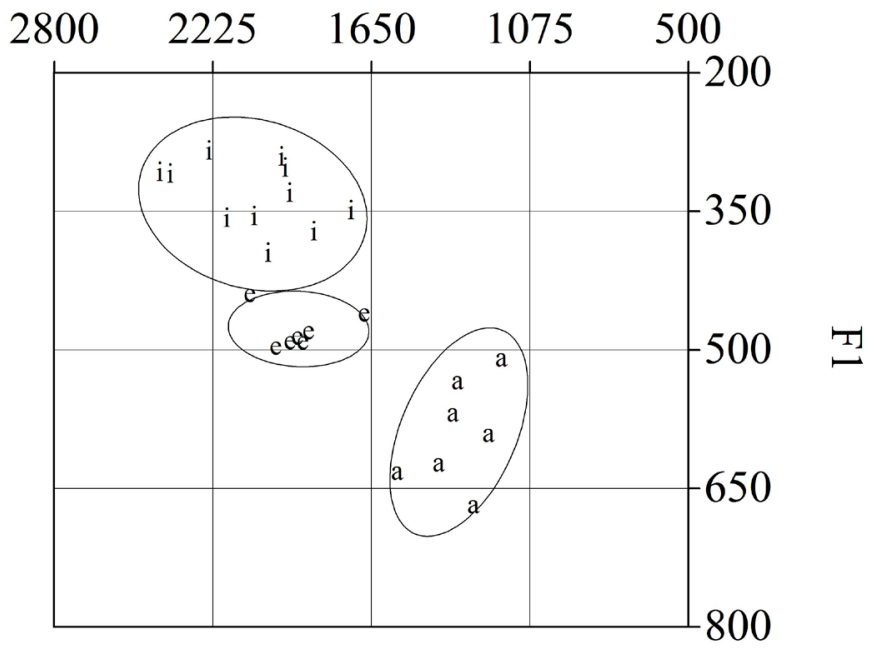

En geometría de rasgos, como en la propuesta de Clements y Hume $(1995)^{5}$, la representación fonética de las vocales atestiguadas tomaría en cuenta el rasgo [abierto] del punto APERTURA y los rasgos [coronal] y [dorsal] para el PUNTO VOCÁLICO (P-V) como se puede observar en (6). En términos de $\mathrm{P}-\mathrm{V}$, las vocales $[\mathrm{u}$ ] y [a] se caracterizarían como [dorsal] mientras que [i] y [e], [coronal]. En relación a Apertura, las vocales [i] y [u] se caracterizan como [-abierto] mientras que [a], [+abierto].

(6) Vocales [i, e, u, a] desde una teoría de rasgos

\begin{tabular}{|l|c|c|c|c|}
\hline & \multicolumn{4}{|c|}{ P-V } \\
\hline \multicolumn{1}{|c|}{ Apertura } & \multicolumn{2}{|c|}{ [coronal] } & \multicolumn{2}{c|}{ [dorsal] } \\
\hline [-abierto], [-abierto] & $\mathrm{i}$ & & & $\mathrm{u}$ \\
\hline [-abierto], [+abierto] & & $\mathrm{e}$ & & \\
\hline [+abierto], [+abierto] & & & $\mathrm{a}$ & \\
\hline
\end{tabular}




\subsection{Contexto de aparición de las vocales monoptongadas}

Las vocales monoptongadas [e]/[e:] e [i]/[i:] son atestiguadas cuando a una raíz nominal que termina en las vocales /a, i, u/ se le añade los marcadores de 1.a y 2.a persona poseedora, como se aprecia en (7). Por una parte, cuando a una raíz que termina en vocal [dorsal] [+abierto] se le afija el marcador de 1.a o 2.a persona poseedora, el segmento resultante es una vocal media [coronal] que puede ser breve [e] o larga [e:]. Por otra parte, cuando a una raíz que termina en vocal [coronal] o [dorsal], pero siempre [-abierto], se le afija el marcador de 1.a o 2.a, el segmento resultante es una vocal alta [coronal] que puede ser breve [i] o larga [i:].

(a)

\begin{tabular}{|c|c|c|}
\hline+ & pers. pos. & $>$ \\
\hline+ & 1.a pers. pos. & $>$ \\
\hline $\mathrm{uNku}+$ & 1.a pers. pos. & $>$ \\
\hline
\end{tabular}

(b)

$\begin{array}{lllll}\text { uma } & + & \text { 2.a pers. pos. } & > & \text { 'u.me.ki } \\ \text { maki }+ & \text { 2.a pers. pos. } & > & \text { 'ma.ki.ki } \\ \text { JuNku }+ & \text { 2.a pers. pos. } & > & \text { 'Jun.gi.ki }\end{array}$

De acuerdo con (7.a), las vocales monoptongadas largas [e:] e [i:] aparecen exclusivamente con el sufijo de 1.a persona poseedora, mientras que las vocales breves [e] e [i] con el sufijo de 2.a persona poseedora. Por ejemplo, en la figura 9, la palabra ['Jun.gi:] </SuNku/+ 1.a pers. pos. 'mi corazón' evidencia el surgimiento de una vocal coronal alargada [i:], que dura aproximadamente 211 ms; mientras que en la 10 , la palabra ['Jun.gi.ki] $</ \int \mathrm{uNku} /+2$.a pers. pos. 'tu corazón' evidencia una vocal breve, que mide 89 ms. 
Jairo Valqui, Gustavo Solís, Carlos Faucet, Franklin Espinoza, Liz Velasquez

Figura 9. Espectrograma de la palabra ['Jun.gi:]

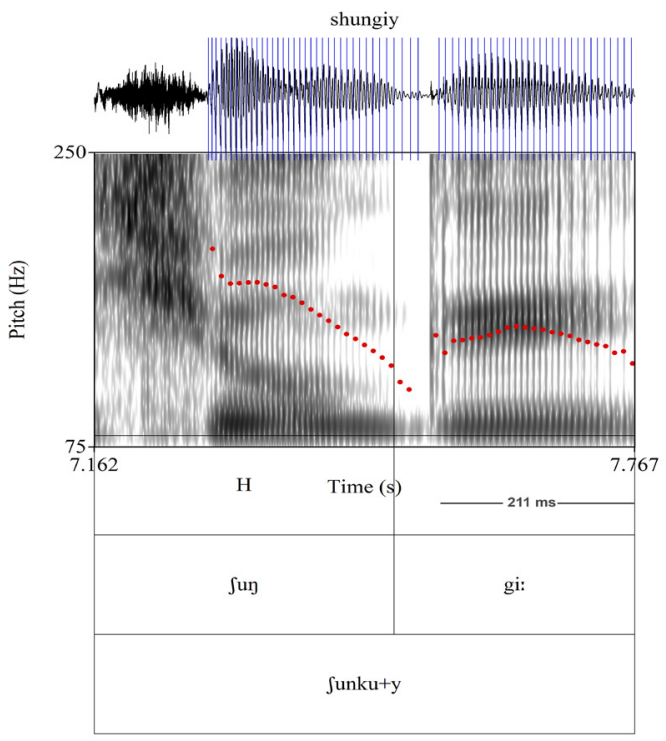

Figura 10. Espectrograma de la palabra ['Jun.gi.ki]

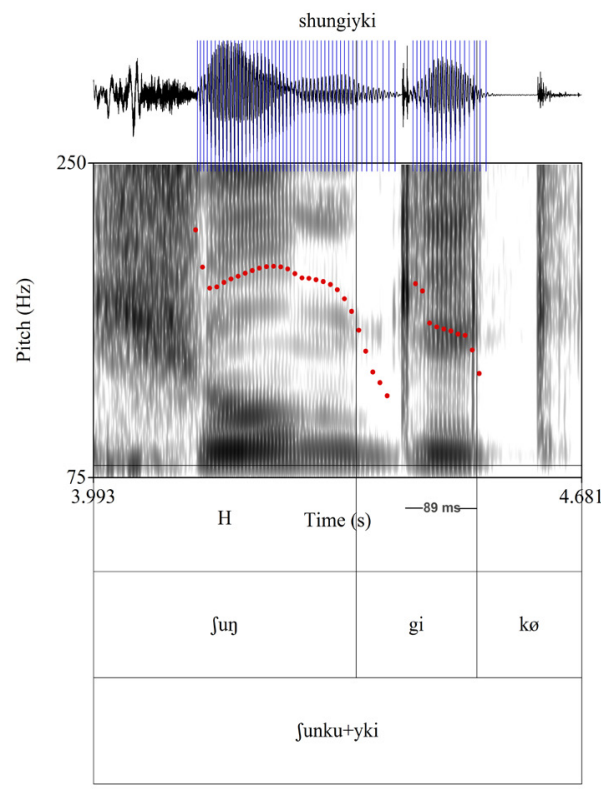




\section{Análisis y discusión}

4.1. Los fenómenos reportados en $\$ 3$ evidencian un proceso de fusión vocálica (monoptongación) en la variedad quechua de Chachapoyas. Los segmentos finales de raíces más el segmento inicial de los sufijos de primera y segunda persona convergen en las vocales monoptongadas [e], [e:], [i] e [i:]. En términos fonológicos, siguiendo a Schane (1995), el proceso descrito se explica en dos dimensiones del segmento melódico: uno donde interviene la apertura representada con $|\mathrm{a}|$ y otro donde interviene la tonalidad, representada con $|\mathrm{y}|$. La apertura y tonalidad se evidencian en (8a) con el surgimiento de una vocal [e:] en esta variedad quechua; y la tonalidad en $(8 b)$ y $(8 c)$ con la aparición de la vocal coronal alargada [i:].

(8)
(a)
$>\mathrm{RR}<$
$\left[\varnothing_{12}, a_{1}, y_{2}\right] \quad /$
[ay] >
[e:]
(b)
$>\mathrm{RR}<$
$\left[\emptyset_{12}, \mathrm{i}_{1}, \mathrm{y}_{2}\right]$
[iy] >
[i:]
(c) $\quad>\mathrm{RR}<$
$\left[\varnothing_{12}, \mathrm{u}_{1}, \mathrm{y}_{2}\right] \quad /$
[uy] >
[i:]

En los tres casos presentados en (8), se observa en realidad que la tonalidad recurrente es, siguiendo la teoría de Schane, un caso de palatalidad, representado por |i|. Esta observación nos permite pensar en una generalización de este proceso de fusión. Se entiende, por un lado, que los rasgos involucrados en el segmento vocálico final de la raíz son [+abierto] para el caso de (8.a) y [-abierto] para el caso de (8.b) y (8.c); y, por otro lado, que el único rasgo involucrado en el punto de constricción para el segmento de los sufijos en cuestión es [coronal], que en este caso corresponde a la "palatalidad" |i|, más el rasgo [-abierto] de la altura vocálica. La generalización de (8) en términos de rasgos nos permite formular una nueva configuración como se presenta en (9).

(9) $>\mathrm{RR}<\quad\left[\varnothing_{12},[ \pm \text { abierto }]_{1}, \begin{array}{r}\left.[\text { coronal }]_{2}\right] \\ {[\text {-abierto }]_{2}}\end{array}\right.$ 
Desde este punto de vista, podemos postular una nueva representación del segmento inicial de la primera y segunda persona poseedora como una unidad subespecificada solo con el rasgo [coronal] y [-abierto] asociada a una unidad de tiempo $(\mu)$, como se representa en R2 en figura 11. La propuesta de un segmento subespecificado es plausible en términos teóricos (cf. Steriade, 1995) y prácticos en la medida que genera el resultado esperado y se apoya en datos objetivos -la ausencia de un segmento pleno [y] en los registros acústicos-.

Figura 11. Representación subespecificada del segmento de la primera y segunda persona poseedora en el quechua de Chachapoyas (R2)

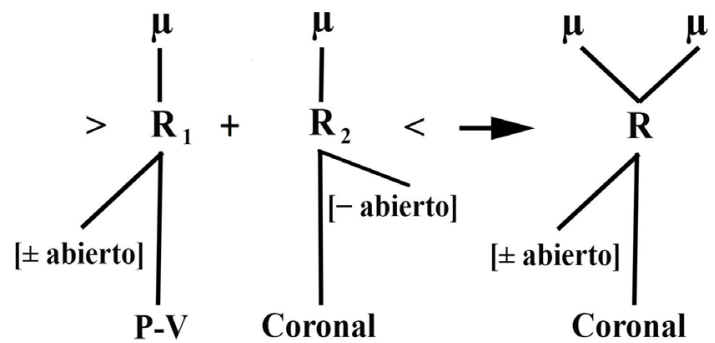

Pero, ¿qué implicancias presentaría esta propuesta de un segmento subespecificado [coronal], [-abierto] en los sufijos de 1.a y 2.a persona poseedora, distinta de la presentada por Taylor como /y/? Lo más interesante de esta reformulación es que reabre una discusión en torno a la forma fonológica de la primera persona poseedora en las variedades modernas del quechua y la evolución de este marcador desde la protolengua. De acuerdo con Cerrón-Palomino (2003), la marca isomórfica de la primera persona en el protoquechua habría sido ${ }^{*} v-y$ (acento en la vocal final de la base, más $-y$ ), que habría provenido del preprotoquechua ${ }^{*}-\mathrm{ya}^{6}$ . Esta forma haría explícita la relación entre el quechua de Pacaraos y las variedades del QI con respecto al acento de la primera persona, que habría surgido a causa de una regla que produce las formas alargadas, atestiguadas en el QI o central. Dentro de este marco, la reformulación de la representación fonológica de la primera persona en el quechua de Chachapoyas permitiría visualizar una posible relación con variedades centrales del QI, como ya había sido prefigurado por Quesada (1988) y ahora es parte de una discusión mayor sobre las relaciones lingüísticas y genéticas de esta variedad quechua con otras cercanas (Cf. Barbieri y otros, 2017). Si se asume que el alargamiento para indicar la primera persona en las variedades del QI central responde a una representación subespecificada radical que solo presenta la unidad de tiempo $(\mu)$ y se evidencia en la duración 
(Cf. Solis, 1996), esta nueva representación guardaría una relación estrecha con la propuesta para el quechua de Chachapoyas, como queda reflejado en la figura 12. El hecho de que se produzca el alargamiento en el QI central y la fusión (monoptongación) en el chachapoyano (QII) se debería justamente a que en estas variedades la representación fonológica de la primera persona poseedora estaría subespecificada como producto de un proceso de debilitamiento paulatino que, iniciado en */-ya/, habría producido formas segmentales completas como /-y/ y formas subespecificadas como /[coronal], [-abierto]/ o solo con la unidad de tiempo $/ \mu /$, dependiendo de cada variedad moderna del quechua.

Figura 12. Propuesta de relación entre variedades del QI con variedades del QII
Q-I
Q-II

\section{Quechuas centrales Quechua de Chachapoyas}
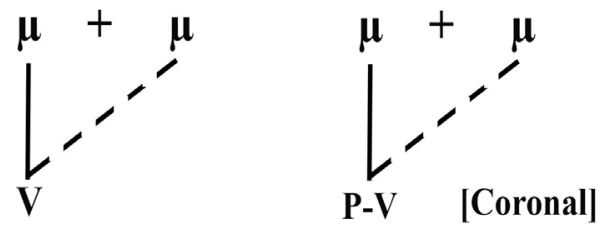

En cuanto al sufijo de 2. ${ }^{\text {a }}$ persona poseedora, datos semejantes a los que presentamos para el chachapoyano han sido reportados en la variedad de Huaylas (Escribens y Proulx, 1970; Parker, 1976; Julca, 2009), donde /urku/ + /-yki/ y /waaka/ + /yki/ dan [ur'kiiki] 'tu frente' y [waa'keeki] 'tu vaca', respectivamente, pero /wayi/ + /yki/ resultan en ['wayiki] 'tu casa'. Con esto se dibuja un patrón interesante: en relación con el marcador de primera, el quechua de Chachapoyas habría sido más conservador que el de Huaylas y las demás variedades de QI; pero en relación con el marcador de segunda, esta vez el quechua de Chachapoyas sería el más innovador. Asimismo, este cuadro reafirmaría la posible relación cercana del chachapoyano con el QI.

4.2. Ahora bien, el proceso de fusión que se ha explicado para el quechua de Chachapoyas con la regla (9) produce las vocales monoptongadas alargadas que dan cuenta de los casos presentados en (7a), pero ¿cómo se explican las formas breves atestiguadas en (7b)? Para Taylor (2000), la alternancia entre vocales monoptongadas breves y alargadas se explicaba como producto de la intervención 
del acento. Este autor señala que las vocales monoptongadas alargadas aparecerán en sílabas acentuadas y las breves en las inacentuadas.

ô, ê e î son largas en palabras monosilábicas o en sílaba acentuada (la primera u otra marcada por un acento para indicar un énfasis emotivo); en la transcripción de los textos, empleamos el acento grave para indicar la combinación de la reducción vocálica y el acento emotivo: mêlòb = mêlôb < /may lado-pi/ “ipor cuál lado (debe encontrarse)!”; ô, ê e î son breves en sílabas no acentuadas. ô y ê se pronuncian cerradas o abiertas según el informante y el contexto. (Taylor, 2000, p. 65)

Sin embargo, los datos acústicos con lo que se cuenta ahora no concuerdan con la explicación del acento como la unidad interviniente en la alternancia entre las vocales monoptongadas breves y alargadas. Estos datos indican que en la variedad quechua de Chachapoyas ambas vocales (breves y alargadas) aparecen en sílabas inacentuadas. Por ejemplo, en la palabra [Simi:] 'mi boca' < / Simi/ + [coronal] [-abierto], el contorno tonal ${ }^{7}$ indica que el acento se ubica en la primera sílaba como se puede observar en la figura 13, como también en las figuras 9 y 10.

Figura 13. El contorno tonal (pitch) en la palabra ['Jimi:]

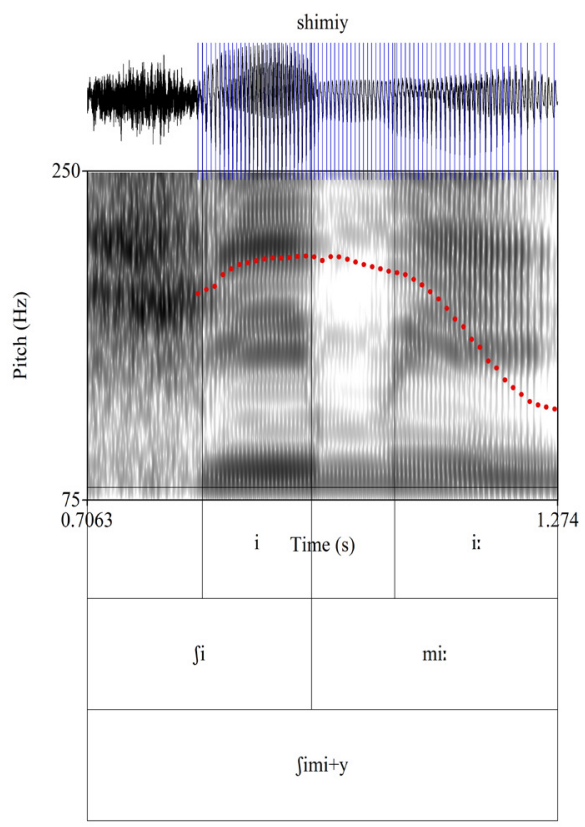


Si la alternancia entre las vocales monoptongadas breves y alargadas no está condicionada por el acento, entonces, ¿qué otra unidad fonológica podría ser la responsable de tal distribución? Un contexto que parece estar relacionado es el límite de palabra (\#) para el caso de las vocales monoptongadas alargadas y la sílaba $(\sigma)$ que sucede a las vocales breves, como se observa en (10):

(10)

$\begin{array}{ll}\text { (a)_\# } & \text { (b)_o } \\ \text { 'ume: } & \text { 'umeki } \\ \text { 'maki: } & \text { 'makiki } \\ \text { 'Jungi: } & \text { 'Jungiki } \\ \text { 'Simi: } & \text { 'Simiki }\end{array}$

Datos del frisón reportados por Graaf y Tiersma (1980) parecen apoyar una solución similar. En esta lengua, que exhibe alternancias entre vocales monoptongadas largas y breves, el acortamiento ocurre cuando la vocal monoptongada está seguida por otra sílaba o por un grupo de consonantes, según se ve en (11).

(11)

$\begin{array}{lll}\text { Sg. } & \text { Pl. } & \\ \text { vi:f } & \text { vifkə } & \text { (small) woman } \\ \text { he:x } & \text { hextə } & \text { high/height } \\ \text { kno:p } & \text { knopkə } & \text { (small) button }\end{array}$

Fuente: Schane (1995, p. 598)

Si el acortamiento de la vocal monoptongada en esta variedad quechua fuese un caso similar al del frisón, tendríamos que preguntarnos por qué no ocurre cuando a las formas de (10a) se les añade el sufijo locativo /-pi/. Por ejemplo, en [ $\left.\int \mathrm{imi}: \beta\right]$ 'en mi boca' </ $\mathrm{jimi}+\mathrm{y}+\mathrm{pi} /$, en donde la vocal monoptongada se mantiene alargada, según está registrado en la figura 14. 
Figura 14. Espectrograma de la palabra $\left[\int \mathrm{imi}: \beta\right]</ \int \mathrm{Simi}+\mathrm{y}+\mathrm{pi} /$

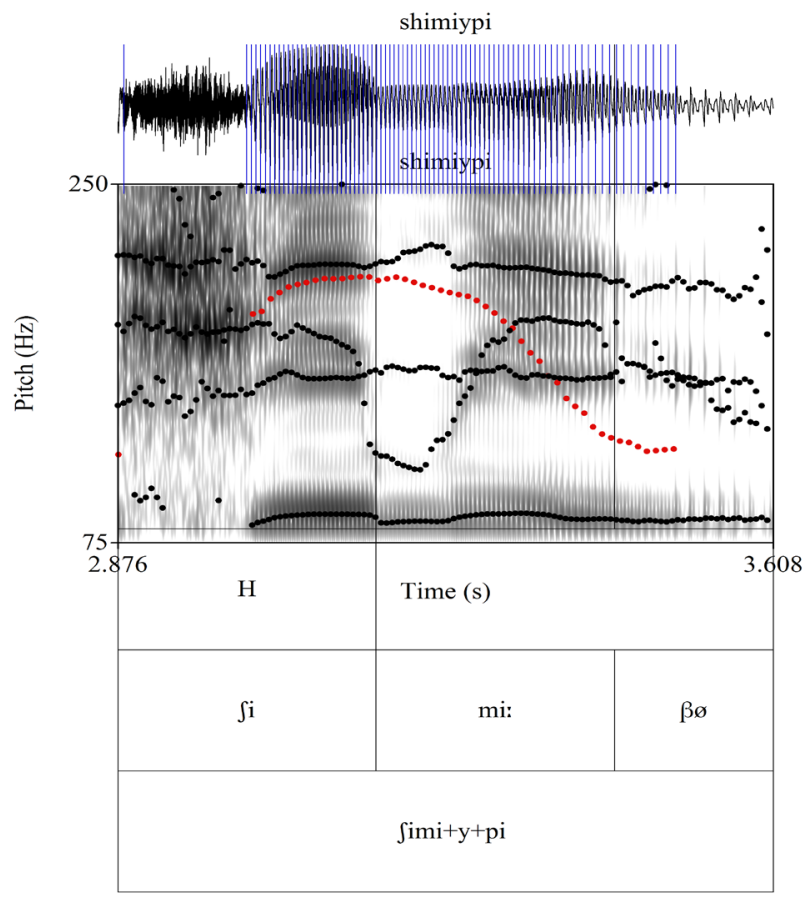

Entonces, si la alternancia de estas vocales breves y largas no es producto de algún contexto fonológico, ¿qué otro nivel de la gramática podría explicar esta distribución? Una hipótesis de trabajo vincula una relación entre la morfología y la fonología en lo que se denomina fonología léxica. En estos términos, el proceso fonológico aquí atestiguado sería dependiente de la información morfológica. Es decir, la monoptongación, como proceso fonológico, interactuaría con las reglas de formación de palabras; en este caso, si a la raíz nominal se le adjunta un sufijo de primera o segunda persona poseedora en la morfología, la base así formada pasaría a la fonología y en ella se producirían las vocales alargadas [V:]. Después de esta operación, las vocales alargadas se harían breves V: > V solo si les sucediese una sílaba, como se representa en (12).

$$
>\mu \mu</ \_\sigma
$$

Como resume Cole (1995), la teoría denominada fonología léxica asume que la salida de cada operación morfológica representa una entrada 
para la aplicación de las reglas fonológicas. De ese mecanismo se deduce que toda regla léxica será cíclica, puesto que interactúa con las reglas de formación de palabras. Si asumimos que, en un primer ciclo, las raíces nominales reciben el sufijo de primera y segunda persona poseedora, en este ciclo se aplicaría la regla propuesta en (9). En un segundo ciclo, estas bases sufrirían un proceso de acortamiento vocálico siempre y cuando se cumpliese la condición de la regla (12) como se puede observar en (13):

\begin{tabular}{|c|c|c|c|}
\hline (a) & & & \\
\hline$[\text { uma }]_{[\text {coronal] }}$ & {$\left[\right.$ maki] $_{[\text {coronal] }}$} & {$\left[\int_{\text {unku }}\right]_{[\text {coronal] }}$} & \\
\hline ume: & maki: & Sunki: & Regla (9) \\
\hline 'u.me: & 'ma.ki: & 'Jun.ki: & Regla (12) \\
\hline
\end{tabular}

\begin{tabular}{|c|c|c|c|}
\hline (b) & & & \\
\hline$\left[\mathrm{uma}_{[\text {coronal] }} \mathrm{ki}\right.$ & {$[\mathrm{maki}]_{[\text {coronal] }} \mathrm{ki}$} & {$\left[\int_{\mathrm{unku}}\right]_{[\text {coronal] }} \mathrm{ki}$} & \\
\hline ume:ki & maki:ki & Junki:ki & Regla (9) \\
\hline 'u.meki & 'ma.kiki & ' Jun.kiki & Regla (12) \\
\hline
\end{tabular}

De acuerdo con esta propuesta, las vocales monoptongadas breves se derivan en la interacción entre la morfología y la fonología cuando a las raíces nominales se les añade el sufijo de segunda persona poseedora. Este proceso morfofonológico evidenciaría que el acortamiento solo ocurre cuando se asigna la segunda persona poseedora según la contextualización de la regla (12). Sincrónicamente, el proceso de fusión (monoptongación) aquí estudiado ocurre tanto en la derivación de la primera y la segunda persona poseedora, y el acortamiento solo en la derivación de la segunda ${ }^{8}$. 


\section{Conclusión}

El proceso de fusión (monoptongación) estudiado ocurre cuando a las raíces nominales se les añade los sufijos de primera y segunda persona poseedora. En términos teóricos, una regla como (9) da cuenta de la fusión y posibilita pensar en un segmento subespecificado solo con el rasgo [coronal], [-abierto] y asociado a una unidad de tiempo $(\mu)$. Esta propuesta develaría una relación estrecha entre el quechua de Chachapoyas -variedad adscrita al QII- y variedades del QI, y reabriría, de esta manera, la discusión en torno a la representación de la primera y segunda personas poseedoras en las variedades modernas del quechua, la forma del marcador en la protolengua, su proceso de cambio histórico y una posible relación entre el chachapoyano y variedades de quechua I. En lo concerniente a las alternancias entre las vocales $[\mathrm{i}] /[\mathrm{i}:]$ y $[\mathrm{e}] /[\mathrm{e}:]$ producidas por este proceso, se demuestra mediante datos acústicos que el acento no explica tal distribución y que, más bien, la interacción entre la morfología y la fonología, dentro de la denominada fonología léxica, explica mejor este caso.

\section{Referencias bibliográficas}

Barbieri, Ch. y otros. (2017). Enclaves of genetic diversity resisted Inca impacts on population history. Scientific Reports 7, Article number: 17411. doi: 10.1038/ s41598-017-17728-w

Cerrón-Palomino, R. (2003). Lingüística quechua [2.a Ed.]. Cusco: Centro de Estudios Rurales Andinos "Bartolomé de Las Casas".

Chaparro, C. (1958). Fonología y lexicón del quechua de Chachapoyas. Lima: Editorial Sagsa.

Clements, G. y Hume, E. (1995). The Internal Organization of Speech Sounds. En Odden, D., y Goldsmith, J., The Handbook of Phonological Theory (pp. 245306). Cambridge/Oxford: Blackwell.

Cole, J. (1995). The Internal Organization of Speech Sounds. En Odden, D., y Goldsmith, J., The Handbook of Phonological Theory (pp. 70-113). Cambridge/ Oxford: Blackwell.

Escribens, A. y Proulx, P. (1970). Gramática del quechua de Huaylas. Lima: UNMSM. 
Heggarty, P. (2005). Enigmas en el origen de las lenguas andinas: aplicando nuevas técnicas a las incógnitas por resolver. Revista Andina, 40, 9-57.

Julca Guerrero, F. (2009). Quechua ancashino. Una mirada actual. Lima: CARE Perú/ Fondo Editorial del Pedagógico San Marcos.

Parker, G. (1976). Gramática quechua Áncash-Huailas. Lima: IEP/MINEDU.

Quesada-Castillo, F. (1988). Phonological Processes in Quechua and Their Implications for Phonological Theory. Tesis doctoral, State University of New York at Buffalo.

Schane, S. (1995). Diphthongization in Particle Phonology. En The Handbook of Phonological Theory. En Odden, D., y Goldsmith, J., The Handbook of Phonological Theory (pp. 586-608). Cambridge/Oxford: Blackwell.

Solís, G. (1996). Los orígenes de la cantidad vocálica en quechua. Documento de Trabajo, 56. Lima: CILA-UNMSM.

Steriade, D. (1995). Underspecification and Markedness. En The Handbook of Phonological Theory. En Odden, D., y Goldsmith, J., The Handbook of Phonological Theory (pp. 112-174). Cambridge/Oxford: Blackwell.

Taylor, G. (2006). Diccionario quechua Chachapoyas Lamas. Lima: IFEA/IEP. (2000). Estudios lingüísticos sobre Chachapoyas. Lima: UNMSM/IFEA.

(1994). Estudios de dialectología quechua (Chachapoyas, Ferreñafe, Yauyos). Lima: UNE.

Torero, A. (2002). Idiomas de los Andes. Lingüística e Historia. Lima: IFEA/Editorial Horizonte.

Unesco (2003). Vitalidad y peligro de desaparición de las lenguas. Documento adoptado por la Reunión Internacional de Expertos sobre el programa de la UNESCO “Salvaguardia de las Lenguas en Peligro". París.

Valqui, J. y Ziemendorff, M. (2016). Vestigios de una lengua originaria en el territorio de la cultura chachapoya. Letras, 87 (125), 5-32.

Weber, D. (1975). Apuntes sobre el quechua de Lamud. SIL. 
${ }^{1}$ Hasta el momento no se han clarificado las circunstancias que explican cómo se instaló esta variedad quechua en el territorio de los chachapoya que, de acuerdo a los estudios de Taylor (2000) y Valqui y Ziemendorff (2016), era un espacio donde se hablaba una lengua distinta a esta lengua andina. Evidencias que reflejan algún momento de una relación entre estas lenguas se perciben en los topónimos y antropónimos que se distinguen en dicha zona.

${ }^{2}$ En los pueblos de Yambajalca-Diosán, Granada, Olleros y Quinjalca en el Alto Imaza (Chachapoyas), Taylor y Chaparro atestiguaron la presencia de un sonido lateral palatal $[\Lambda]$ en contextos en los que en los demás pueblos de Chachapoyas y Luya aparece una africada palatal [d3] (Taylor, 1994) o una fricativa palatal [3] (Weber, 1974, p. 5).

${ }^{3}$ Como se verá a lo largo del trabajo, en el contexto de los marcadores de personas poseedoras del quechua chachapoyano no habría un diptongo que se monoptongue sincrónicamente. Por ello, utilizamos la etiqueta "monoptongación" de Taylor solo por economía y tradición, excepto cuando nos situamos en el marco de la teoría de Schane, donde la etiqueta en cuestión tiene el sentido técnico de fusión de partículas o rasgos (cf. $\$$ 1), uso al que sí nos adosamos.

${ }^{4}$ Taylor utiliza el circunflejo en ê y î para indicar que estas vocales se han monoptongado.

${ }^{5}$ En la teoría de rasgos de Clements y Hume (1995), la altura vocálica se representa a través del rasgo [abierto] que depende del nudo Apertura. Además, para este marco teórico las vocales pueden representarse con los rasgos de constricción [labial], [coronal] y [dorsal].

${ }^{6}$ Según señala Cerrón-Palomino, "el presupuesto general en el que se basa la reconstrucción de *-ya es la hipótesis, refrendada en muchísimos casos, de que los sufijos de persona no son sino una copia reducida de los respectivos lexemas pronominales" (2003, p. 141). El lexema pronominal en cuestión estaría evidenciado en la sílaba inicial del pronombre yaqa de primera persona de cierta variedad wanka.

${ }^{7} \mathrm{El}$ contorno tonal parece ser uno de los correlatos fonéticos del acento más estables en la variedad quechua de Chachapoyas.

${ }^{8}$ Desde una perspectiva histórica, Quesada (1988) propone que el proceso de monoptongación empezó en la secuencia $\mathrm{i}+\mathrm{y}$ de la marca de la segunda persona poseedora -yki, que dio como resultado, en un primer momento, formas como ${ }^{\star}$ wasi:ki. Luego, las entradas con [i:] se habrían acortado en [i]. 